University of Nebraska - Lincoln

DigitalCommons@University of Nebraska - Lincoln

7-1998

\title{
Measurement of the photorefractive grating phase shift in a polymer by an ac phase-modulation technique
}

\author{
Martin M Liphardt \\ University of Nebraska - Lincoln \\ Stephen Ducharme \\ University of Nebraska-Lincoln, sducharme1@unl.edu
}

Follow this and additional works at: https://digitalcommons.unl.edu/physicsducharme

Part of the Physics Commons

Liphardt, Martin M and Ducharme, Stephen, "Measurement of the photorefractive grating phase shift in a polymer by an ac phase-modulation technique" (1998). Stephen Ducharme Publications. 81.

https://digitalcommons.unl.edu/physicsducharme/81

This Article is brought to you for free and open access by the Research Papers in Physics and Astronomy at DigitalCommons@University of Nebraska - Lincoln. It has been accepted for inclusion in Stephen Ducharme Publications by an authorized administrator of DigitalCommons@University of Nebraska - Lincoln. 


\title{
Measurement of the photorefractive grating phase shift in a polymer by an ac phase-modulation technique
}

\author{
Martin Liphardt and Stephen Ducharme \\ Department of Physics and Astronomy, Center for Materials Research and Analysis, University of Nebraska, \\ Lincoln, Nebraska 68588-0111
}

Received September 16, 1997; revised manuscript received November 17, 1997

The complex coupling constant of a photorefractive polymer was measured as a function of an applied electric field by use of a modified ac phase modulation technique. We determined that both a photorefractive index grating and a nonphotorefractive absorption grating were present. The electric field dependencies of the amplitude and the phase of the photorefractive gain coefficient were accurately described by standard photorefractive theory. The accuracy of the photorefractive phase shift as measured by this phase-modulation technique was $\pm 1^{\circ}$ near phase shifts of $90^{\circ}$ and $\pm 3^{\circ}$ near phase shifts of $45^{\circ}$. (C) 1998 Optical Society of America [S0740-3224(98)01807-4]

OCIS codes: $160.5320,160.5140,160.5470$.

\section{INTRODUCTION}

The photorefractive phase shift is a measure of the spatial shift of a photorefractive index grating with respect to the light interference fringes that form the grating. The phase shift depends on the details of charge transport and charge trapping, on the grating formation conditions and history, and on material properties. Therefore, investigating the photorefractive phase shift will provide useful information about the transport and trapping properties of a particular photorefractive material. These types of investigation are also suited to differentiating the contributions from any nonphotorefractive gratings (such as absorption gratings) that may be present. ${ }^{1,2}$ This differentiability is of utmost importance because contributions from any nonphotorefractive grating can cause misinterpretation of coupling or diffraction data and may lead to incorrect conclusions.

In recent years a number of techniques based on twobeam energy coupling have been proposed to measure the photorefractive phase shift. In these techniques a phase shift between the writing beams is introduced by use of a ramped electro-optic modulator, ${ }^{3}$ a piezoelectric ac phase modulator, ${ }^{4-9}$ or an electro-optic ac phase modulator. ${ }^{10,11}$ Other techniques use Bragg cells to introduce small frequency shifts (heterodyne detection) ${ }^{12-15}$ or make use of the differences of (phase-sensitive) two-beam coupling and simple (phase-insensitive) diffraction. ${ }^{16,17}$ Monitoring the two-beam coupling signal when rapidly translating the grating (or the sample) can also be a method to measure the photorefractive phase shift. ${ }^{18-21}$

In this paper we present results of the measurement of the photorefractive phase shift in the photorefractive polymer bisphenol A 4,4' -nitroaminostilbene (bisA-NAS) with 29 wt.\% benzaldehyde diphenyl hydrazone ${ }^{22}(\mathrm{DEH})$, using a modified ac phase-modulation technique based on the method introduced by Cudney et al. ${ }^{11}$

\section{THEORY AND METHOD}

The technique employed to measure the photorefractive phase shift follows the approach of Cudney et al. ${ }^{11}$ in which phase modulation is imposed on one of the coupling beams by means of an electro-optic modulator. We have chosen to induce phase modulation by using a piezoelectric mirror with linear operation over the desired range. The piezoelectric mirror method has been used in a number of ac modulation techniques. ${ }^{5,7}$

To analyze the data we modified the two-beam coupling analysis of Cudney et al. ${ }^{11}$ to include bulk absorption and to account for the slanted grating geometry needed for two-beam coupling in photorefractive polymers. Consider two plane waves that are incident upon the sample with the sample normal in the $z$ direction (see Fig. 1): $\mathbf{E}_{1}(\mathbf{x}, t)=\hat{e}_{1} E_{1}(z, t) \exp \left[-i\left(\mathbf{k}_{1} \cdot \mathbf{x}-\omega t\right)\right]$ and $\mathbf{E}_{2}(\mathbf{x}, t)$ $=\hat{e}_{2} E_{2}(z, t) \exp \left[-i\left(\mathbf{k}_{2} \cdot \mathbf{x}-\omega t\right)\right]$, where $\hat{e}_{1,2}$ are the unit polarization vectors, $\mathbf{k}_{1,2}$ are the wave vectors, and $E_{1,2}$ are the amplitudes of the pump and probe beams, respectively. Note that we explicitly allow the amplitude of the two beams to be time dependent to account for the phase modulation of the probe beam. The interference pattern produced by these two beams may give rise to an index grating by means of the photorefractive effect and to both index and absorption gratings by means of, e.g., photochromic effects. We consider only gratings with amplitudes proportional to the modulation depth (or fringe contrast) $m(z, t)=2 E_{1}(z, t) E_{2} *(z, t) / I_{0}(z, t)$ of the interference pattern, where $I_{0}(z, t)$ $=E_{1}(z, t) E_{1} *(z, t)+E_{2}(z, t) E_{2} *(z, t)$, so in general the complex index of refraction $\tilde{n}$ can be written as

$$
\begin{aligned}
\tilde{n}= & n_{0}+n_{1} \cos \left(\mathbf{K} \cdot x+\phi_{n}\right) \\
& +i\left[\kappa_{0}+\kappa_{1} \cos \left(\mathbf{K} \cdot x+\phi_{\kappa}\right)\right],
\end{aligned}
$$

where the grating wave vector $\mathbf{K}=k_{2}-k_{1}, n_{0}$ and $\kappa_{0}$ 


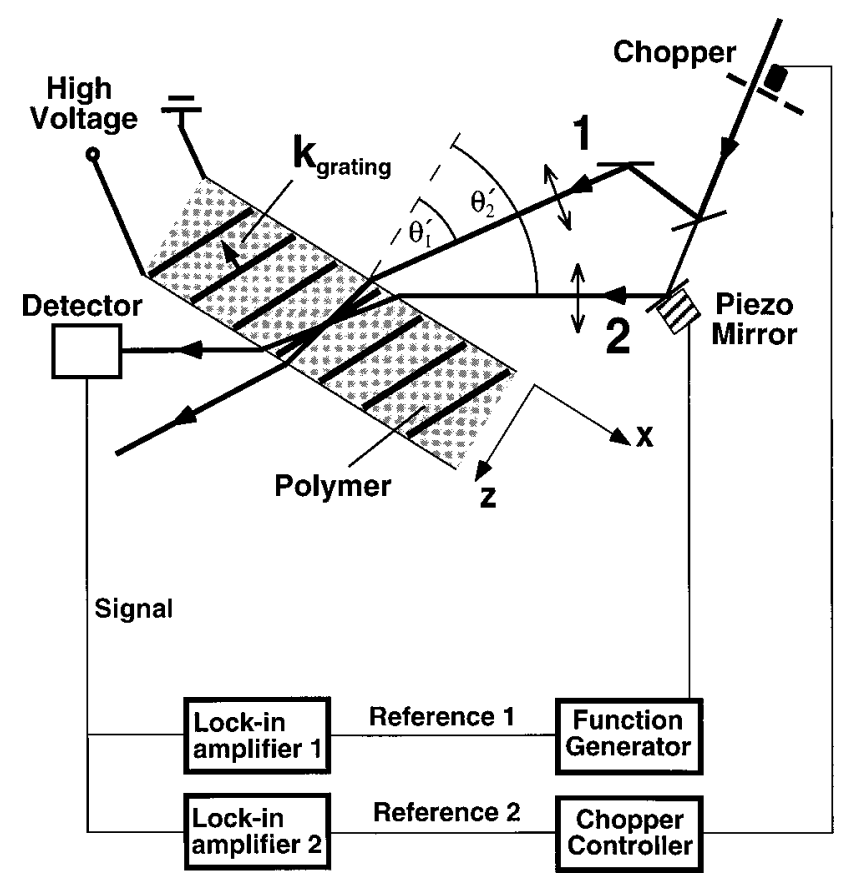

Fig. 1. Experimental setup to measure the photorefractive phase shift. The glass plates of the sample are omitted for clarity.

are the real and the imaginary (absorption) parts of the uniform index of refraction, $n_{1}$ and $\kappa_{1}$ are the amplitudes of the index and the absorption gratings, and $\phi_{n}$ and $\phi_{\kappa}$ are the phase shifts of the index and absorption gratings with respect to the interference pattern, respectively.

The slowly varying amplitude approximation of the coupled-wave analysis yields the following set of equations ${ }^{23}$ :

$\cos \theta_{1} \frac{\mathrm{d} E_{1}(z, t)}{\mathrm{d} z}=-\frac{\Gamma_{1}}{4}\left\langle m^{*}(z, t)\right\rangle_{t} E_{2}(z, t)-\frac{\alpha}{2} E_{1}(z, t)$,

$\cos \theta_{2} \frac{\mathrm{d} E_{2}(z, t)}{\mathrm{d} z}=-\frac{\Gamma_{2}}{4}\langle m(z, t)\rangle_{t} E_{1}(z, t)-\frac{\alpha}{2} E_{2}(z, t)$,

where the complex coupling constant $\Gamma_{1,2}$ $=i 4 \pi\left[n_{1} \exp \left(\mp i \phi_{n}\right)+i \kappa_{1} \exp \left(\mp i \phi_{\kappa}\right)\right] / \lambda, \theta_{1,2}$ are the internal angles of incidence of the pump and the probe beams, respectively, the subscript $t$ refers to the timeaveraged value, and $\alpha=4 \pi \kappa_{0} / \lambda$ is the absorption coefficient. Note that we have allowed for absorption gratings that are phase shifted from the interference pattern $\left(\phi_{\kappa}\right.$ $>0$ ), as is often the case for population gratings. ${ }^{2}$ We have assumed that the phase modulation of the probe beam amplitude $E_{2}(z, t)$ occurs at a rate that is much too fast for all the gratings to respond, or $\Omega \tau \gg 1$, where $\Omega$ is the modulation frequency and $\tau$ is the response time of the fastest grating. Therefore steady-state gratings arewritten at the time-averaged value of the modulation depth $\langle m\rangle_{t}$. Solutions to Eqs. (2) are readily obtained in the undepleted-pump approximation $[m(z, t) \ll 1$ for 0 $<z<L]$. The solutions will account for bulk absorp- tion and grating curvature and will also be applicable to the slanted grating geometry.

The phase modulation of the probe beam can be expressed as $E_{2}(0, t)=E_{2}(0) \exp [i \mu \sin (\Omega t)]$, where $\mu$ is the (small) phase-modulation amplitude. The steadystate harmonic components of the intensity of the exiting probe beam at temporal frequencies $0, \Omega$, and $2 \Omega$ are given by ${ }^{11}$

$$
\begin{aligned}
I_{2}[0] & =I_{20} \exp \left(\Gamma_{R} L^{\prime}\right), \\
I_{2}[\Omega] & =2 \mu I_{20} \exp \left(\Gamma_{R} L^{\prime} / 2\right) \sin \left(\Gamma_{I} L^{\prime} / 2\right), \\
I_{2}[2 \Omega] & =\frac{\mu^{2}}{2} I_{20}\left[\exp \left(\Gamma_{R} L^{\prime} / 2\right) \cos \left(\Gamma_{I} L^{\prime} / 2\right)-1\right],
\end{aligned}
$$

where $\Gamma_{R}\left(\Gamma_{I}\right)$ refers to the real (imaginary) part of the complex coupling constant, $I_{20}$ is the intensity of the probe beam in the absence of beam coupling, and $L^{\prime}$ is the effective sample thickness $\left[L^{\prime}=L / \cos \left(\theta_{2}\right)\right]$. A function generator supplied the ac voltage at a frequency of $\Omega=260 \mathrm{~Hz}$ to drive the piezoelectric mirror and the reference signal for lock-in amplifier 1, as shown in Fig. 1. We used this lock-in amplifier to measure the first harmonic $\left(I_{2}[\Omega]\right)$ and the second harmonic $\left(I_{2}[2 \Omega]\right)$ (one at a time). To improve the signal-to-noise ratio of dc component $I_{2}[0]$ we used a mechanical chopper and lock-in amplifier 2 (Fig. 1) operating at $2000 \mathrm{~Hz}$ so the amplitude modulation would not interfere with the operation of the first lock-in amplifier.

We use Eqs. (3)-(5) to determine the modulation amplitude $\mu$ and the real and imaginary parts of the complex coupling constant $\Gamma$, a total of three unknowns. This is in contrast to the method of Cudney et al. ${ }^{11}$ who performed an independent measurement of the modulation amplitude. Such an ex situ calibration has the disadvantage that external parameters such as temperature may change the calibration, resulting in an incorrect value for the modulation amplitude. The coupling of the probe and the pump beams constitutes an interferometer in itself, which permits the in situ measurement of the modulation amplitude. To increase the accuracy of the measurement further, a series of data was collected at different values of the modulation amplitude $\mu$, which was proportional to the amplitude $V$ of the ac voltage applied to the piezoelectric transducer (in the transducer's linear operating range). Plotting both $I_{2}[\Omega]$ and $I_{2}[2 \Omega]$ as a function of the ac piezoelectric transducer voltage $V$ and performing a linear (for $I_{2}[\Omega]$ ) and a quadratic (for $I_{2}[2 \Omega]$ ) curve fit resulted in an accurate measurement of $\Gamma_{I}$, the imaginary part of the complex coupling constant.

In the absence of any gratings other than the photorefractive grating, the photorefractive phase shift $\phi_{\mathrm{PR}}$ can be computed by use of its definition:

$$
\tan \phi_{\mathrm{PR}}=\Gamma_{R} / \Gamma_{I} .
$$

In general, though, absorption gratings and nonphotorefractive index gratings may also be present. In this case the real and imaginary components of the complex cou- 
pling constant contain information not only about the photorefractive grating but also about the other gratings. It is necessary to measure the real and imaginary components of the coupling constants, $\Gamma_{1}$ and $\Gamma_{2}$, obtained from measurements of the pump and probe beam harmonic components with the aid of Eqs. (3)-(5). The four unknowns, $n_{1 R}, n_{1 I}, \phi_{R}$, and $\phi_{I}$, are obtained from the coupled-wave theory as summarized in Table 1.

From Table 1 it is apparent that a measurement of the complex coupling constant as seen by the pump beam $\left(\Gamma_{1}\right)$ and as seen by the probe beam $\left(\Gamma_{2}\right)$ would provide the necessary information to solve for all four unknowns by taking sums and differences of the results. In principle one can accomplish this goal by adding a second detector to measure simultaneously the intensity of the pump beam. In this case, though, one may not work in the undepleted-pump regime, because in that approximation the pump beam intensity is not affected by beam coupling. One can easily argue that using probe and pump beams of approximately equal intensity $(m \approx 1)$ will result in the highest sensitivity. However, using probe and pump beams of approximately equal intensity is undesirable because this would make the beam coupling analysis much more complicated ${ }^{7,21,24}$ (even without the phase modulation) and would also lead to pronounced higher harmonics of the space-charge field..$^{25,26}$

As an alternative to measuring the gain as seen by both the probe beam and the pump beam, one can use two different two-beam coupling geometries in such a way that in one of the geometries no contribution from the photorefractive effect is allowed (because of symmetry arguments). ${ }^{11}$ This technique yields two complex coupling constants, one that is due to nonphotorefractive contributions only and one that is due to both photorefractive and nonphotorefractive contributions.

Another technique for obtaining two distinct complex coupling constants is based on physically reversing the direction of what is commonly referred to as the $c$ axis of the sample (the axis that breaks the inversion symmetry, a necessary condition for a nonzero electro-optic effect). This procedure is equivalent to measuring the complex coupling constant as seen by the probe beam and then by the pump beam, with the exception that one can still work in the undepleted-pump regime. The disadvantage of this technique and the technique introduced by Cudney et $a l .{ }^{11}$ is that one actually measures the coupling constant in two different instances; the sample is physically moved between measurements. The potential exists for introducing errors because of optical misalignments or other external parameters.

Many photorefractive polymers possess the feature that the breaking of the inversion symmetry of these

Table 1. Contributions to the Complex Coupling Constant $\Gamma$ when Both Probe and Pump Beams Are Monitored

\begin{tabular}{cccccc}
\hline & \multicolumn{2}{c}{$\Gamma_{R}($ Real Part) } & & $\Gamma_{I}$ (Imaginary Part) \\
\cline { 2 - 3 } \cline { 5 - 6 } Grating Type & Index & Absorption & & Index & Absorption \\
\hline$\Gamma_{1}$ & $+n_{1} \sin \phi_{n}$ & $-\kappa_{1} \cos \phi_{\kappa}$ & $+n_{1} \cos \phi_{n}$ & $+\kappa_{1} \sin \phi_{\kappa}$ \\
$\Gamma_{2}$ & $-n_{1} \sin \phi_{n}$ & $-\kappa_{1} \cos \phi_{\kappa}$ & $+n_{1} \cos \phi_{n}$ & $-\kappa_{1} \sin \phi_{\kappa}$ \\
\hline
\end{tabular}

Table 2. Contributions to the Complex Coupling Constant $\Gamma$ for Both Directions $(+,-)$ of the Applied Electric Field

\begin{tabular}{clc}
\hline $\begin{array}{c}\text { Coupling } \\
\text { Constant }\end{array}$ & $\begin{array}{c}\text { Contributions to } \\
\Gamma_{R}(\text { Real Part })\end{array}$ & $\begin{array}{c}\text { Contributions to } \\
\Gamma_{I} \text { (Imaginary Part) }\end{array}$ \\
\hline$\Gamma_{+}$ & $+n_{\mathrm{PR}} \sin \phi_{\mathrm{PR}}$ & $+n_{\mathrm{PR}} \cos \phi_{\mathrm{PR}}$ \\
& $-\kappa_{\mathrm{POP}} \cos \phi_{\mathrm{POP}}$ & $+\kappa_{\mathrm{POP}} \sin \phi_{\mathrm{POP}}$ \\
& $-\kappa_{\mathrm{PC}} \operatorname{lin} \phi_{\mathrm{PR}}$ & $+n_{\mathrm{PR}} \cos \phi_{\mathrm{PR}}$ \\
$\Gamma_{-}$ & $-n_{\mathrm{PR}} \sin \cos \phi_{\mathrm{POP}}$ & $-\kappa_{\mathrm{POP}} \sin \phi_{\mathrm{POP}}$ \\
& $-\kappa_{\mathrm{POP}} \cos$ \\
& $-\kappa_{\mathrm{PC}}$ & \\
\hline
\end{tabular}

amorphous materials is performed in situ by means of an externally applied electric field (the dipoles of the polymer partially align under the influence of the electric field). Consequently one can reverse the direction of poling by simply changing the sign of the applied electric field. By measuring the complex coupling constant for both negative and positive applied electric fields we can independently determine the amplitude and the phase of the photorefractive index grating and an absorption grating. This method is reliable because there is no need to move the sample, optical beams, or detectors. The analysis of the data is based on the assumption that only three types of grating are present (see Table 2): a photorefractive index grating (including contributions from related orientational gratings ${ }^{27}$ ) with amplitude $n_{\mathrm{PR}}$ and phase $\phi_{\mathrm{PR}}$, a population absorption grating with amplitude $\kappa_{\mathrm{POP}}$ connected with the photorefractive space charge and having the same phase $\phi_{\mathrm{POP}}=\phi_{\mathrm{PR}}$, and an in-phase absorption grating (e.g., photochromic) with amplitude $\kappa_{\mathrm{PC}}$ and phase $0^{\circ}$ that is independent of the electric field or depends only on even powers of the electric field. (Mechanisms that yield such gratings are discussed below.) Note that this assumption reduces the number of possible contributions from eight (index versus absorption grating, local versus nonlocal grating, and odd versus even in electric field) to four, which is the maximum number of independent contributions that this method can distinguish. The complex coupling constant, both real and imaginary parts, is measured twice [with the help of Eqs. (3)-(5)]: $\Gamma_{+}=\Gamma_{+R}+i \Gamma_{+I}$ for one direction of the applied electric field and $\Gamma_{-}=\Gamma_{-R}+i \Gamma_{-I}$ for the other. The four parameters $n_{\mathrm{PR}}, \kappa_{\mathrm{POP}}, \kappa_{\mathrm{PC}}$, and $\phi_{\mathrm{PR}}$ can then be determined from the following relations:

$$
\begin{aligned}
1 / 2\left(\Gamma_{+R}-\Gamma_{-R}\right) & =n_{\mathrm{PR}} \sin \left(\phi_{\mathrm{PR}}\right), \\
1 / 2\left(\Gamma_{+R}+\Gamma_{-R}\right) & =\kappa_{\mathrm{POP}} \cos \left(\phi_{\mathrm{POP}}\right)+\kappa_{\mathrm{PC}}, \\
1 / 2\left(\Gamma_{+I}+\Gamma_{-I}\right) & =n_{\mathrm{PR}} \cos \left(\phi_{\mathrm{PR}}\right), \\
1 / 2\left(\Gamma_{+I}-\Gamma_{-I}\right) & =\kappa_{\mathrm{POP}} \sin \left(\phi_{\mathrm{POP}}\right) .
\end{aligned}
$$

\section{RESULTS AND DISCUSSION}

We measured the complex coupling constant of a sample of the photorefractive polymer compound bisA-NAS with 29 wt. \% DEH. A 50- $\mu$ m-thick film was formed between glass plates coated with transparent indium tin oxide electrodes by techniques described in detail elsewhere. $^{22,28}$ The absorption coefficient at $650 \mathrm{~nm}$ was 
$245 \mathrm{~cm}^{-1}$, and the index of refraction was $1.72 \pm 0.02$ as measured by spectroscopic ellipsometry. ${ }^{29}$ The angles of incidence of the two $p$-polarized writing beams (polarized in the plane of Fig. 1) were $60^{\circ} \pm 0.2^{\circ}$ and $40^{\circ} \pm 0.2^{\circ}$, respectively, and the ratio of the probe beam (beam 2 ) to the pump beam (beam 1 ) intensities was $\beta=1 / 40$ at a total light intensity of approximately $1 \mathrm{~W} / \mathrm{cm}^{2}$ from a cw $\mathrm{TEM}_{00}$ mode $\mathrm{Ar}^{+}$-pumped dye laser operating at $650 \mathrm{~nm}$. These parameters resulted in a grating spacing $\Lambda=2.60$ $\pm 0.05 \mu \mathrm{m}$ with the grating wave vector oriented at $63.6^{\circ} \pm 0.6^{\circ}$ from the sample normal. We measured the complex coupling constant as a function of the applied voltage over the range $0-3000 \mathrm{~V}(0-600 \mathrm{kV} / \mathrm{cm})$. The phase-modulation frequency was set to $260 \mathrm{~Hz}$, and the phase-modulation amplitude $\mu$ was varied from approximately 0.01 to $0.2 \mathrm{rad}$. These experimental conditions satisfy the key approximations, low intensity modulation $m$ and low phase-modulation amplitude $\mu$, made in the two-beam coupling analysis presented above. The amplitudes of the photorefractive and nonphotorefractive coupling constants obtained from Eqs. (7) are plotted in Fig. 2 as a function of the applied electric field component along the grating wave vector. At high electric fields the photorefractive (index) grating dominates, but at lower fields the strength of the absorption grating is comparable with that of the photorefractive index grating. The sign of the absorption grating was such that both beams experienced loss, whereas the photorefractive gain coefficient (for probe beam 2 in Fig. 1) was positive for negative applied voltages. The nonphotorefractive absorption grating shows only a weak dependence on the electric field. (This is in fact our primary evidence that the absorption grating is not connected with the photorefractive space charge. In that case there would be a pronounced dependence of the modulated trap occupation, and hence of the absorption grating amplitude, on the applied electric field.) The presence of a substantial absorption grating is not surprising considering the large absorption coeffi-

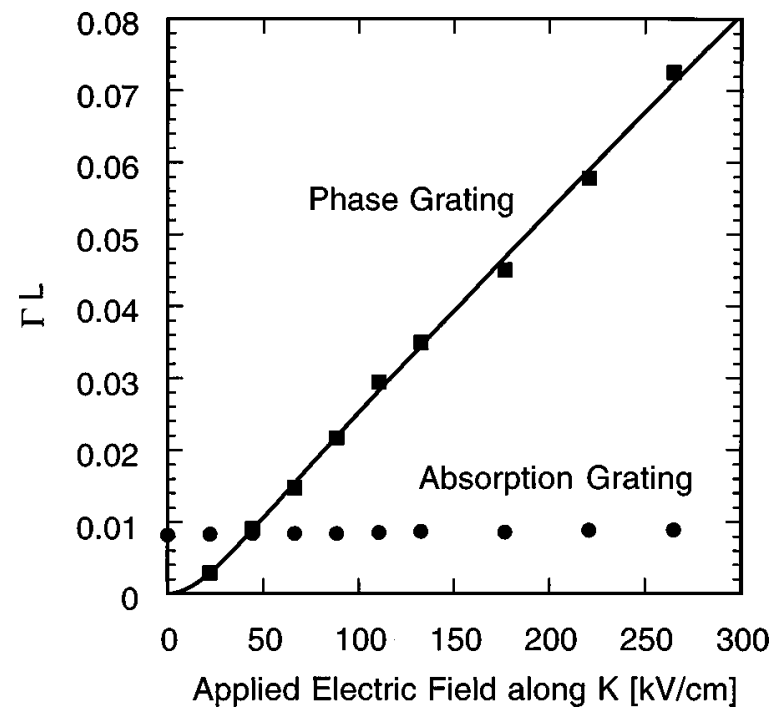

Fig. 2. Amplitude of the coupling strength $\Gamma L$ coefficient from index $\left(\Gamma_{R}\right)$ and absorption $\left(\Gamma_{I}\right)$ gratings as a function of the applied electric field strength along the grating wave vector. The solid curve is a least-squares fit of the index grating gain to Eq. (8). cient of this photorefractive polymer and the possibility of photoinduced cis-trans isomerization of the nitroaminostilbene (NAS) chromophore.

A first test of whether the index grating is primarily photorefractive is to fit the electric field dependence of the photorefractive gain coefficient $\Gamma_{\mathrm{PR}} \sim E_{0} E_{s c}$ to standard photorefractive theory (neglecting photovoltaic effects), ${ }^{30}$ where

$$
E_{\mathrm{sc}}=m E_{q}\left[\frac{E_{0}{ }^{2}+E_{d}^{2}}{E_{0}{ }^{2}+\left(E_{d}+E_{q}\right)^{2}}\right]^{1 / 2}
$$

where $E_{0}=(V / L) \sin \left[\left(\theta_{2}+\theta_{1}\right) / 2\right]$ is the component of the applied electric field along the grating wave vector [see Fig. 1 and Eqs. (2)]. It is this component that affects photorefractive space-charge accumulation. Photorefractive polymer films are typically studied in a slanted geometry to tilt the grating wave vector out of the film plane and partially along the applied field.

We achieved good agreement without relying on modifications to the analysis of Ref. 30 that would take into consideration the pronounced electric field dependence of the charge-carrier generation efficiency and mobility ${ }^{31-33}$ that has been reported for polymeric systems. ${ }^{34,35}$ For this fit we assume that the effective electro-optic coefficient is proportional to the applied electric field, ${ }^{36,37}$ as is appropriate for a sample that poles at operating temperature and may also include contributions from orientational gratings. ${ }^{27}$ In this geometry we calculate the diffusion field $E_{d}=k_{B} T K / e=0.62 \pm 0.01 \mathrm{kV} / \mathrm{cm}$, where $K=2 \pi / \Lambda$. We obtained the limiting space-charge electric field $E_{q}=40 \pm 4 \mathrm{kV} / \mathrm{cm}$ by fitting the data in Fig. 2 to Eq. (8). This number is approximately a factor of 5 smaller than the number previously measured for other samples of the same photorefractive polymer composite, ${ }^{37}$ possibly a consequence of $\mathrm{DEH}$ crystallization with age ${ }^{22}$ in this 1-month-old sample.

We and others have been successful in applying ${ }^{24,36-39}$ the standard model ${ }^{30,40}$ of photorefractive charge transport to polymers for which the processes of charge generation, transport, and trapping, although they are microscopically different from those in crystalline materials, can be described macroscopically by the familiar generation rate, mobility, and trapping rate expressions. ${ }^{30,40}$ In contrast to these qualitative similarities, there are several important quantitative distinctions between organic and inorganic photorefractive materials. The relatively low dielectric constants in organics result in significantly higher limiting space-charge fields. ${ }^{36,37}$ Photorefractive polymers are routinely studied with applied fields $E_{0}$ approaching or exceeding $E_{q}$ because polymer samples tend to be thin enough and have high enough dielectric strength to permit application of external fields up to several million volts per centimeter. The electro-optic response in low- $T_{g}$ polymers is proportional to the applied field because the polar nonlinear optical molecules are oriented by the field. This makes the grating amplitudes much more strongly dependent on the applied field. ${ }^{36,37}$ An interesting consequence of this is the discovery of orientational enhancement ${ }^{27}$ leading to spectacular improvements in the two-beam energy coupling gain. ${ }^{37,41}$ 
To test the assumption that the index grating is of photorefractive origin and the absorption grating is not, the real and imaginary components of the index grating and the absorption grating are plotted in Fig. 3 as a function of the component of the applied electric field along the grating wave vector. The index grating is strongly field dependent, and the absorption grating is not. The real (in-phase) part of the absorption grating is independent of the strength of the applied electric field within the experimental uncertainty. This grating may be connected with photochromic changes of the absorption coefficient or with photoinduced cis-trans isomerization of the NAS chromophore. We can expect that these changes will be in phase with the interference fringes. The small imaginary part of the absorption grating $\left(\phi=90^{\circ}\right)$ exhibits a pronounced electric field dependence and may be due to trapped charges, a population grating associated with the photorefractive grating. $^{2}$ However, a fit to the data by use of the same electric field dependence of the amplitude and phase as for the photorefractive index grating did not produce a good fit for electric fields below $50 \mathrm{kV} / \mathrm{cm}$, and the parameters of the fit would result in a field dependence of what was earlier interpreted as a possibly photochromic grating. A population grating is still a reasonable candidate to explain the data because shallow traps may cause the phase of the population grating $\left(\phi_{\mathrm{POP}}\right)$ to be different from the phase of the photorefractive grating $\left(\phi_{\mathrm{PR}}\right)$. Unfortunately, the experiment cannot separate each contribution to the real part of the absorption grating, which is necessary for determining the phase of the population grating. [Also note that the experiment is insensitive to any nonlocal (or phase-shifted) contributions that are either independent of the electric field or depend only on even powers of the electric field.]

The final test of the photorefractive origin of the index grating is to compare the phase shift with the predictions of photorefractive theory. The phase shift of the index grating [defined in Eq. (6)] is plotted in Fig. 4 as a function of the component of the applied electric field along

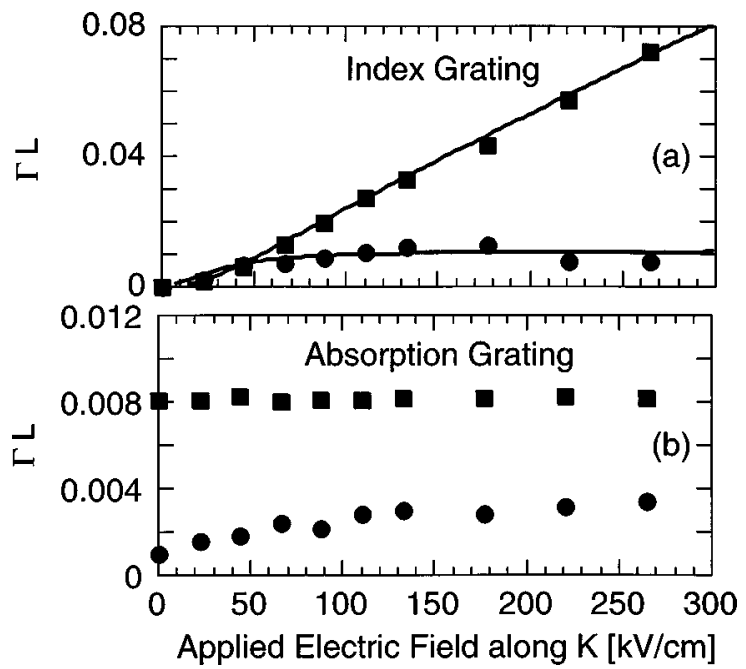

Fig. 3. Real (squares) and imaginary (circles) parts of (a) the index grating and of (b) the absorption grating as a function of the applied electric field strength along the grating wave vector. The solid curves are least-squares fits of the index grating gain to Eqs. (8) and (9).

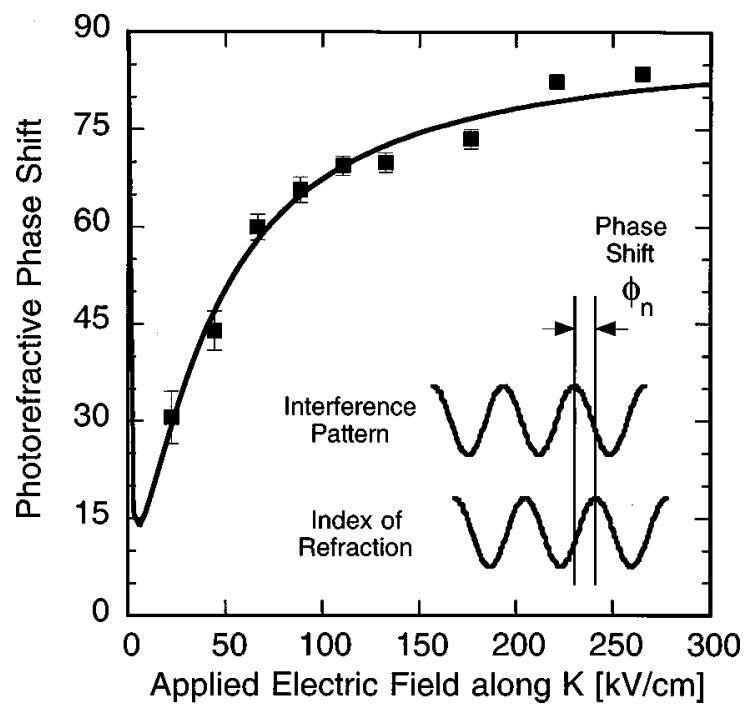

Fig. 4. Phase shift of the index grating as a function of the applied electric field strength along the grating wave vector. The solid curve is a least-squares fit to Eq. (9).

the grating wave vector. The phase shift at the lowest measured applied electric field is approximately $30^{\circ}$ and increases steadily, saturating near $90^{\circ}$ as the electric field increases. The signal strength scales with electric field because the electro-optic coefficient is proportional to the electric field, and at applied electric fields below $\sim 25 \mathrm{kV} / \mathrm{cm}$ the signal becomes too small to be reliably measured. The solid curve is a least-squares fit to the expression for the photorefractive grating phase (neglecting photovoltaic effects) ${ }^{30}$ :

$$
\tan \phi_{\mathrm{PR}}=\frac{\operatorname{Im}\left(\mathbf{E}_{\mathrm{sc}}\right)}{\operatorname{Re}\left(\mathbf{E}_{\mathrm{sc}}\right)}=\frac{E_{d}\left(E_{d}+E_{q}\right)+E_{0}{ }^{2}}{E_{0} E_{q}} .
$$

Again, the behavior can be described by standard photorefractive theory. From the curve fit we determine the limiting space-charge electric field to be $E_{q}=42 \pm 2$ $\mathrm{kV} / \mathrm{cm}$. This number is in good agreement with the value ( $E_{q}=40 \pm 4 \mathrm{kV} / \mathrm{cm}$ ) obtained from the curve fit of the amplitude plotted in Fig. 4. From the limiting spacecharge electric field we determined the effective density of photorefractive traps to be $N_{\mathrm{pr}}=2.0 \pm 0.2 \times 10^{15} \mathrm{~cm}^{-3}$. The relatively low trap density compared with that of the sample from Ref. $37\left(N_{\mathrm{pr}}=1.5 \pm 0.5 \times 10^{16} \mathrm{~cm}^{-3}\right)$ is responsible for the lower overall gain obtained in this sample, because maximum gain is proportional to the limiting space-charge electric field $E_{q}$. The sign of the phase shift depends on the intrinsic orientation of the sample polar axis; in the low- $T_{g}$ polymers this direction is controlled by the applied field.

\section{CONCLUSIONS}

We have presented experimental results of the measurement of the complex coupling constant of the photorefractive polymer bisA-NAS:DEH as a function of an applied electric field. The coupling constant was measured by a modified ac phase-modulation technique. We determined that both a photorefractive index grating and a nonphotorefractive absorption grating were created by 
the writing beams. The absorption grating likely was produced by two different mechanisms, trapped charges and photochromic effects or photoinduced cis-trans isomerization of the NAS chromophore. The electric field dependence of the photorefractive gain coefficient was accurately described by standard photorefractive theory. The phase-modulation technique provides improved accuracy in measurement of the photorefractive phase shift (compared with the commonly used sample translation technique ${ }^{20}$ ) of $\pm 1^{\circ}$ near phase shifts of $90^{\circ}$ and $\pm 3^{\circ}$ near phase shifts of $45^{\circ}$. From fits to the data we determined the limiting space-charge electric field of this polymer to be $41 \pm 3 \mathrm{kV} / \mathrm{cm}$ and the effective density of photorefractive traps to be $2.0 \pm 0.2 \times 10^{15} \mathrm{~cm}^{-3}$. The standard expressions for the magnitude [Eq. (8)] and the phase [Eq. (9)] of the photorefractive steady-state space-charge field successfully describe beam coupling experiments without the need for modifications ${ }^{39}$ to take into account the field dependence of the charge-carrier quantum efficiency, mobility, or lifetime. ${ }^{31-33}$

\section{ACKNOWLEDGMENTS}

This study was supported by the U.S. Air Force Office of Scientific Research, and by the Nebraska Research Initiative through the Center for Materials Research and Analysis. We thank J. M. Takacs and L. Zhang for supplying the bisA-NAS polymer.

\section{REFERENCES AND NOTES}

1. R. B. Bylsma, D. H. Olson, and A. M. Glass, "Photochromic gratings in photorefractive materials," Opt. Lett. 13, 853 (1988).

2. R. S. Cudney, R. M. Pierce, G. D. Bacher, and J. Feinberg, "Absorption gratings in photorefractive crystals with multiple levels," J. Opt. Soc. Am. B 8, 1326 (1991).

3. M. Z. Zha, P. Amrhein, and P. Günter, "Measurement of phase shift of photorefractive gratings by a novel method," IEEE J. Quantum Electron. 26, 788 (1990).

4. P. A. M. D. Santos, L. Cescato, and J. Frejlich, "Interference-term real-time measurement for selfstabilized two-wave mixing in photorefractive crystals," Opt. Lett. 13, 1014 (1988).

5. P. M. Garcia, L. Cescato, and J. Frejlich, "Phase-shift measurement in photorefractive holographic recording," J. Appl. Phys. 66, 47 (1989).

6. P. N. Ilinykh, O. P. Nestiorkin, and B. Ya. Zel'dovich, "Phase shift in nondegenerate coupling of waves in the photorefractive crystal," Opt. Commun. 80, 249 (1991).

7. R. Hofmeister, A. Yariv, A. Kewitsch, and S. Yagi, "Simple methods of measuring the net photorefractive phase shift and coupling constant," Opt. Lett. 18, 488 (1993).

8. R. K. Ing and J.-P. Monchalin, "Measurement of the photorefractive phase shift by polarization interferometry," Opt. Lett. 18, 852 (1993).

9. J. Frejlich, "Real-time photorefractive hologram phase-shift measurement and self-diffraction effects," Opt. Commun. 107, 260 (1994).

10. M. Gehrtz, J. Pinsl, and C. Bräuchle, "Sensitive detection of phase and absorption gratings: phase-modulated, homodyne detected holography," Appl. Phys. B 43, 61 (1987).

11. R. S. Cudney, G. D. Bacher, R. M. Pierce, and J. Feinberg, "Measurement of the photorefractive phase shift," Opt. Lett. 17, 67 (1992).

12. R. M. Montgomery and M. R. Lange, "Amplitude and phase measurement technique for photorefractive gratings," J. Appl. Phys. 68, 4782 (1990).

13. W. B. Lawler, C. J. Sherman, and M. G. Moharam, "Direct measurement of the amplitude and the phase of photorefractive fields in $\mathrm{KNbO}_{3}: \mathrm{Ta}$ and $\mathrm{BaTiO}_{3}$," J. Opt. Soc. Am. B 8, 2190 (1991).

14. P. N. Ilinykh, O. P. Nestiorkin, and B. Ya. Zel'dovich, "Recording a static hologram with laser beams of different frequencies in photorefractive crystals," J. Opt. Soc. Am. B 8, 1042 (1991).

15. D. G. Gray, M. G. Moharam, and T. M. Ayres, "Heterodyne technique for the direct measurement of the amplitude and phase of photorefractive space-charge fields," J. Opt. Soc. Am. B 11, 470 (1994).

16. V. Kondilenko, V. Markov, S. G. Odulov, and M. Soskin, "Diffraction of coupled waves and determination of phase mismatch between holographic grating and fringe pattern," Opt. Acta 26, 239 (1979).

17. R. Kapoor, M. Moghbel, and P. Venkateswarlu, "Energytransfer measurement and determination of the phase shift between the holographic grating and the fringe pattern in photorefractive materials," Opt. Lett. 18, 696 (1993).

18. V. Markov, S. Odulov, and M. Soskin, "Properties of the three-dimensional phase holograms in activated and reduced $\mathrm{LiNbO}_{3}$ crystals," Bull. Acad. Sci. USSR Phys. Ser. 41, 144 (1977).

19. K. Sutter and P. Günter, "Photorefractive gratings in the organic crystal 2-cyclooctylamino-5-nitropyridine doped with 7,7,8,8-tetracyanoquinodimethane," J. Opt. Soc. Am. B 7, 2274 (1990).

20. C. A. Walsh and W. E. Moerner, "Two-beam coupling measurements of grating phase in a photorefractive polymer," J. Opt. Soc. Am. B 9, 1642 (1992).

21. A. Grunnet-Jepsen, C. I. Thompson, and W. E. Moerner, "Measurement of the spatial phase shift in high-gain photorefractive materials," Opt. Lett. 22, 874 (1997).

22. B. E. Jones, S. Ducharme, A. Goonesekera, M. Liphardt, R. V. Athalye, L. Zhang, and J. M. Takacs, "Photoconductivity and grating response time of a photorefractive polymer," J. Opt. Soc. Am. B 11, 1064 (1994).

23. H. Kogelnik, "Coupled wave theory for thick hologram gratings,” Bell Syst. Tech. J. 48, 2909 (1969).

24. M. Liphardt, A. Goonesekera, S. Ducharme, J. M. Takacs, and L. Zhang, "Effect of beam attenuation on photorefractive grating erasure," J. Opt. Soc. Am. B 13, 2252 (1996).

25. N. V. Kukhtarev, "Kinetics of hologram recording and erasure in electro-optic crystals," Sov. Tech. Phys. Lett. 2, 438 (1976).

26. P. Günter and F. Micheron, "Photorefractive effects and photocurrents in $\mathrm{KNbO}_{3}: \mathrm{Fe}$," Ferroelectrics 18, 27 (1978).

27. W. E. Moerner, S. M. Silence, F. Hache, and G. C. Bjorklund, "Orientationally enhanced photorefractive effect in polymers," J. Opt. Soc. Am. B 11, 320 (1994).

28. A. Goonesekera, M. Liphardt, S. Ducharme, J. M. Takacs, and L. Zhang, "The role of photoconductivity in molecularly doped photorefractive polymers," in Xerographic Photoreceptors and Photorefractive Polymers, S. Ducharme and P. M. Borsenberger, eds., Proc. SPIE 2526, 109 (1995).

29. Measured by P. Snyder by means of a J. A. Woollam Company spectroscopic ellipsometer.

30. N. V. Kukhtarev, V. B. Markov, S. G. Odulov, M. S. Soskin, and V. L. Vinetskii, "Holographic storage in electrooptic crystals. I. Steady state," Ferroelectrics 22, 949 (1979).

31. A. Twarowski, "Geminate recombination in photorefractive crystals,” J. Appl. Phys. 65, 2833 (1989).

32. J. S. Schildkraut and A. V. Buettner, "Theory and simulation of the formation and erasure of space-charge gratings in photoconductive polymers," J. Appl. Phys. 72, 1888 (1992).

33. J. S. Schildkraut and Y. Cui, "Zero-order and first-order theory of the formation of space-charge gratings in photoconductive polymers," J. Appl. Phys. 72, 5055 (1992).

34. L. B. Schein, "Comparison of charge transport models in molecularly doped polymers," Philos. Mag. B 65, 795 (1992).

35. J. C. Scott, L. T. Pautmeier, and W. E. Moerner, "Photoconductivity studies of photorefractive polymers," J. Opt. Soc. Am. B 9, 2059 (1992).

36. S. Ducharme, R. W. Twieg, J. C. Scott, and W. E. Moerner, 
"Observation of the photorefractive effect in a polymer," Phys. Rev. Lett. 66, 1846 (1991).

37. M. Liphardt, A. Goonesekera, B. E. Jones, S. Ducharme, J. M. Takacs, and L. Zhang, "High performance photorefractive polymers," Science 263, 367 (1994).

38. W. E. Moerner and S. Silence, "Polymeric photorefractive materials," Chem. Rev. 94, 127 (1994).

39. S. Ducharme, "Applicability of the band transport (Kukhtarev) model to photorefractive polymers," in Xerographic Photoreceptors and Photorefractive Polymers, S.
Ducharme and P. M. Borsenberger, eds., Proc. SPIE 2526, 144 (1995).

40. N. V. Kukhtarev, V. B. Markov, S. G. Odulov, M. S. Soskin, and V. L. Vinetskii, "Holographic storage in electrooptic crystals. II. Beam coupling-light amplification," Ferroelectrics 22, 961 (1979).

41. K. Meerholz, B. L. Volodin, Sandalphon, B. Kippelen, and N. Peyghambarian, "A photorefractive polymer with high optical gain and diffraction efficiency near 100\%," Nature 371, 497 (1994). 